\title{
Deterministic chaos at the ocean surface: applications and interpretations
}

\author{
A. J. Palmer, C. W. Fairall and R. A. Kropfli \\ NOAA Environmental Technology Laboratory, 325 Broadway, Boulder, CO 80303
}

Received: 24 February 1997 - Accepted: 9 June 1998

\begin{abstract}
Ocean surface, grazing-angle radar backscatter data from two separate experiments, one of which provided coincident time series of measured surface winds, were found to exhibit signatures of deterministic chaos. Evidence is presented that the lowest dimensional underlying dynamical system responsible for the radar backscatter chaos is that which governs the surface wind turbulence. Block-averaging time was found to be an important parameter for determining the degree of determinism in the data as measured by the correlation dimension, and by the performance of an artificial neural network in retrieving wind and stress from the radar returns, and in radar detection of an ocean internal wave. The correlation dimensions are lowered and the performance of the deterministic retrieval and detection algorithms are improved by averaging out the higher dimensional surface wave variability in the radar returns.
\end{abstract}

\section{Introduction}

Signatures of deterministic chaos in grazing angle radar backscatter from the ocean surface have been reported for several independent experiments (Haykin, and $\mathbf{L i}$, 1995; Leung and Lo, 1993; and, Palmer, et.al., 1995). This process is far from simple, involving shadowing, diffraction, specular reflection, and other processes not operating at the smaller incidence angles. While there has been recent progress in understanding and modeling grazing angle radar backscatter from the ocean surface (Wetzel, 1990), this effort continues to characterize the dynamics of the process statistically rather than deterministically. A dynamical model relating these processes to surface winds and stress is not known. However, the reports of deterministic chaos occurring simultaneously in surface winds and ocean surface radar backscatter (Palmer, et.al, 1995) indicates that such a dynamical

Correspondence to: A.J. Palmer model exists, and may be reducible to as few as six nonlinearly coupled first-order differential equations for the time scales of those studies. From such a model, response functions and transfer function could, in principle, be derived for radar detection and retrieval applications. In lieu of knowing such a model, in this work we use a trained artificial neural network to simulate these functions. We apply the neural net only in the temporal domain to search for dynamical patterns in data obtained at a fixed location. This distinguishes our work from previous works which have applied neural nets to the retrieval problem in other domains, e.g. (Thiria, et al, 1993). Performance measures of the neural net algorithms and estimates of the dynamical attractor dimension for the data lead us to the hypothesis that the surface wind dynamical system is responsible for the low dimensional signatures in the grazing angle radar backscatter time series.

The first observation of deterministic chaos in seasurface, grazing-angle radar backscatter was reported in Leung and Haykin, 1990. These authors followed up their findings with an important practical application: the development of a deterministic algorithm for radar detection of small ice burgs within a background of radar sea clutter (Haykin, and Li, 1995; Leung and Lo, 1993). Their detection algorithm was based on a nonlinear response function for the sea clutter generated by a trained artificial neural network. Below we demonstrate an identical procedure for radar detection of ocean internal waves within a sea-clutter background which was found to be chaotic, paying particular attention to the effect of time-averaging of the data as a probe of the low dimensional determinism present in the sea-clutter.

In the other important observation of deterministic chaos in sea-surface grazing-angle radar backscatter (Palmer, et.al, 1995), coincident time series measurements of the ocean surface winds were found to exhibit the same signature of deterministic chaos (correlation dimension) as the radar backscatter. This finding 
suggested the obvious interpretation that the low dimensional dynamical system responsible for the radar backscatter determinism is the marine boundary layer turbulent winds that induce the surface structure that produces the radar backscatter. Support for this hypothesis was found in the observation that the vertically polarized (VV) radar backscatter showed signatures of deterministic chaos while the horizontally polarized (HII) backscatter did not (Palmer, et.al., 1995). $\mathrm{VV}$-polarized radar images of sea surface, although not at grazing incidence, are known to more faithfully reveal boundary layer wind structures while $\mathrm{HH}$ - polarized images are more revealing of the ocean surface longwave structures (Smirnov and Zavorotny, 1995). (As mentioned below, this polarization dependence is not always present, evidently depending on sea-state.) In Sect. 3 , we conduct further investigations of this hypothesis, again using time averaging of the data as a probe of the low dimensional determinism present in the data.

The reason that time averaging is a uscful filter for those investigations is that the measurements do not probe an isolated, low-dimensional dynamical system. If they did, time averaging of the data, being a linear transformation of the embedding coordinates cannot alter the dynamical attractor dimension (Abarbanel, et. al, 1993). Random system noise such as the radar receiver noise does not appear to be an important noise source for these studies (signal-to-noise ratios are at least $20 \mathrm{~dB}$ for the time series data utilized). However, the long surface-wave variability mentioned above is evidently a source of dynarnical noise with higher dimension than the surface winds (Elgar and Mayer-Kress, 1989; and Frison and Abarbanel, 1997). We will show some evidence that time averaging of the radar signal can reduce the intrusion of this high- dimensional variability in the data. On the other hand, there is also evidence that shorter sampling times are necessary to reduce the indicated dynamical attractor dimension for the vertical wind time series, and we will offer a possible explanation for this.

We stress that the neural network retrieval and detection algorithms are employed here as a probe of the determinism present in the data, and are not yet proposed as an established method for practical detection and retrieval applications. However, the performance results for the neural network algorithms, particularly their dependence on averaging time for the data, should serve as a guide to future development of robust deterministic retrieval and detection algorithms for grazing angle radar backscatter over the ocean.

\section{Internal Wave Detection}

Radar detection of features on or near the sea surface is hampered by sea clutter, which is the temporal variabil- ity imposed on the backscatter by motions of the surface scattering elements. If the variability of the sea-clutter background is low dimensional, then it may be better isolated from the desired signal by processing its temporal variability with a deterministic algorithm rather than with statistical moments. This has been demonstrated for radar detection of sea ice within a background of sea clutter (Haykin and Li, 1995; and Leung and Lo, 1993). Here, we produce a similar demonstration for deterministic radar detection of ocean internal waves (IWs). As in that work, our study is a comparative evaluation of the performance of a deterministic vs. statistical algorithrn for treating the background, and we demonstrate that a deterministic detection algorithm, using prediction error for the radar return as the estimator, outperforms a statistical regression algorithm using this same estimator. We conduct our evaluation as a function of block-averaging time for the time series of radar returns. We show that the improvement over statistical processing occurs for all of the averaging times examined $(0.2$ - $20 \mathrm{~s}$ ). We also show that the performance of the deterministic detection algorithm is associated with lower cstimates of the dynamical attractor dimension for the background, and this suggests that the improved detection is due, in part, to the low dimensional nature of the sea clutter dynamics.

\subsection{CORE Ocean Sensing Experiment}

The $\mathrm{x}$-band radar backscatter data utilized for this portion of the analysis was obtaincd during a comprehensive multi-sensor experiment on air-sea interactions held off the coast of northern Oregon during September and October of 1995 (Kropfli and Clifford, 1996). The experiment, called the Coastal Ocean Probing Experiment (COPE) utilized the NOAA Environmental Technology Laboratory's two shore-based microwave radars (one at $\mathrm{x}$-band and the other at ka-band) and a full complement. of in situ and other remote sensors, many of which were mounted on the Scripps Institute Floating Instrument Platform (em FLIP). The northern coast of Oregon was selected for its wide variety of atmospheric and oceanic conditions and particularly for the frequency and intensity of tidally forced internal waves (IWs) in that area. The IW packets in the experimental area are forced by tidal flow over a well-defined shelf break about $70 \mathrm{kmn}$ offshore in highly stratified water produced from the nearby Columbia River outflow.

The radars were mounted on a site $744 \mathrm{~m}$ in elevation within $4 \mathrm{~km}$ of the shoreline. For this study, the $\mathrm{x}$-band radar was operated in a staring mode, illuminating a sector of the ocean surface in which FLIP was moored, about $30 \mathrm{~km}$ offshore. A single range gate with a range extent of $150 \mathrm{~m}$ and an azimuthal extent of $500 \mathrm{~m}$ was selected. A five-hour time series of both vertically polarized (VV) and horizontally polarized (HH), normalized radar cross section (NRCS) for this range gate were 


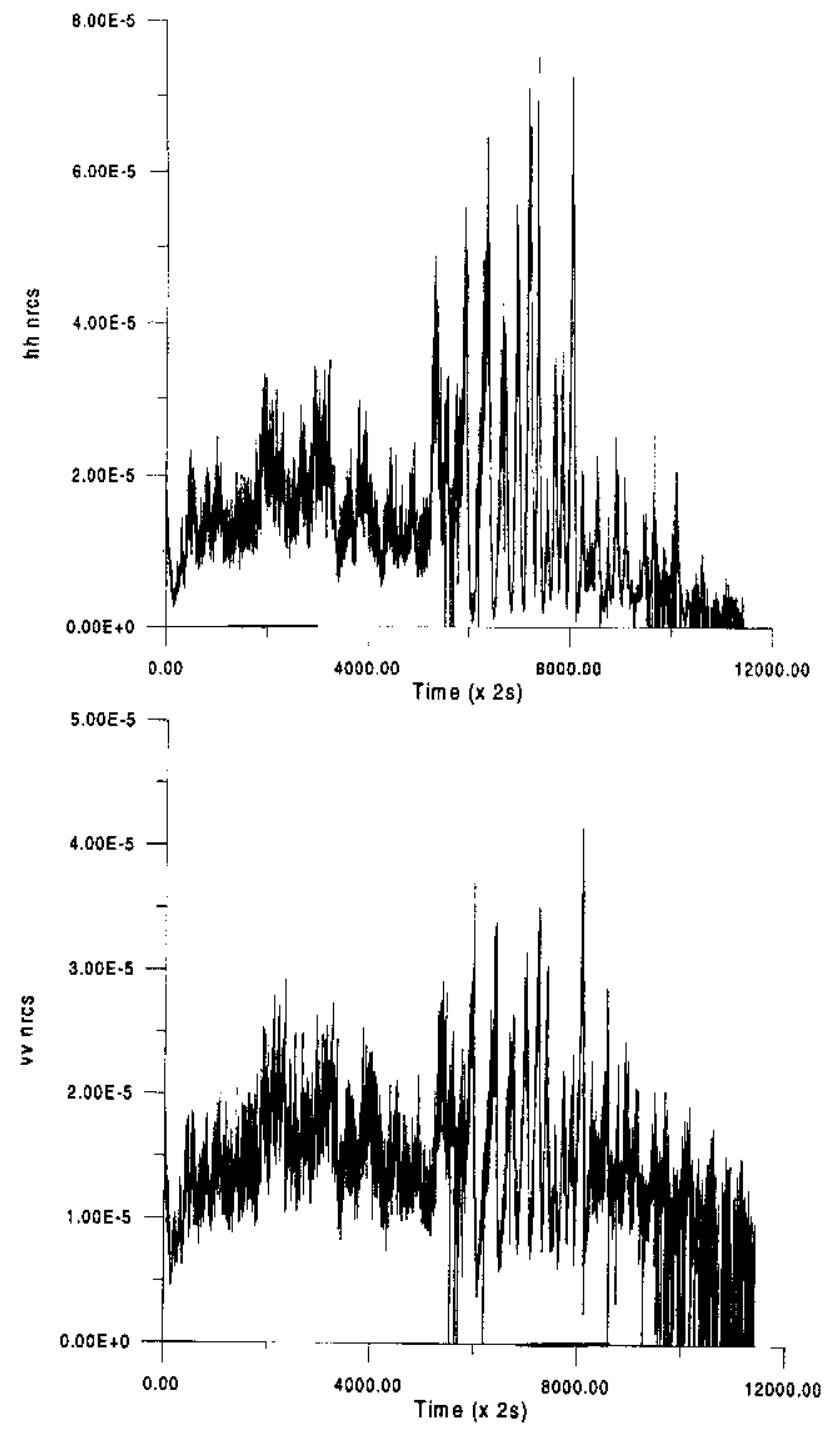

Fig. 1. Time series of 2-s averaged, fixed-beam radar backscatter samples for a single range gate on the ocean surface for VV and $\mathrm{HH}$ polarizations, showing the arrival of the IW packet near the 6000 sample point.

recorded at a $4-\mathrm{Hz}$ sampling rate. The 2 -s averaged, NRCS fixed-beam time series are presented in Fig. 1 showing the arrival of the IW packet near sample number 6000 . Radar images of an IW packet that preceded the fixed-beam data are shown in Fig. 2. As has been previously observed at lower incidence angles (Mityagina, et.al., 1991), the HH-polarized time series and HHpolarized radar innage exhibit the greater contrasi for the IW packet. The winds during the fixed-bcam data averaged $9 \mathrm{~m} / \mathrm{s}$ from the north, which was parallel to the IW wavefronts. The leading edge of the IW packet, as viewed with the radar, propagated at about $0.9 \mathrm{~m} / \mathrm{s}$ and had a crest-to-crest distance near the leading cdge of about $800 \mathrm{~m}$.

\subsection{Correlation Dimension}

Deterministic detection algorithms are expected to exhibit the greatest improvement over statistical algorithms when the dimension of the dynamical attractor governing the background is small, i.e., when there are few significant degrees of freedom participating in the dynamics governing the background variability. Therefore, following Haykin and $\mathrm{Li}, 1995$, and Heung and Lo, 1993, we parallel our tests of deterministic detection of IWs with an estimate of the dynamical attractor dimension for the background portion of the time series. For this purpose, we compute the correlation dimension for the background by applying the Grassburger Procaccia algorithm (Grassburger and Procaccia, 1983) to the portion of the radar backscatter time series before the arrival of the internal wave packet. In order for the correlation dimension to be a valid estimate of the global dynamical attractor dimension, a number of usually unattainable criteria must be met in sampling the time series. For practical purposes however, there is usually a trade- off in the choice of minimum time lag used in the sampling for a fixed record length. Shorter time lags provide a greater number of points with which to sample the attractor, but they do so at the expense of sampling only a local volume of the attractor responsible for all of the motions. Our detection algorithms are based on the local properties of the dynamical attractor and we make no claims as to the invariance of the deterministic algorithm across the longer time scale trends exhibited by the data. We utilize the same number of samples (1000) in all of the correlation dimension computations, recalling that the principal effect of increasing the number of samples is a smaller embedding dimension for the onset of saturation (Ding, et.al, 1993).

A plot of the correlation dimension vs. the embedding dimension for the portion of the HH-polarized, radar backscatter time series before the arrival of the IW packet is shown in Fig. 3 for three different block-averaging times for the data with time delays equal to the blockaveraging time. As seen, when the correlation dimension did show a saturation, its value was less than six. Thus, we chose to fix the order of our detection algorithms (number of input sequential values of the time series used to predict a future value) at six (Elsner and Tsonis, 1992). For the 0.2-s averaging time, the correlation dimension failed to exhibit a saturation, and this result will be used below to help in the interpretation of the detection results.

Unlike an ea rlier nonlinear dynamics study of sea surface radar backscatter (Palmer, et.al, 1995) where the $\mathrm{HH}$ signal was found to have a higher correlation dimension than the VV signal, the HII and VV signals in this data set were found to have essentially the same correlation dimensions and detection performance characteristics. Thus, here and in what follows, we present results only for the HIII data. 


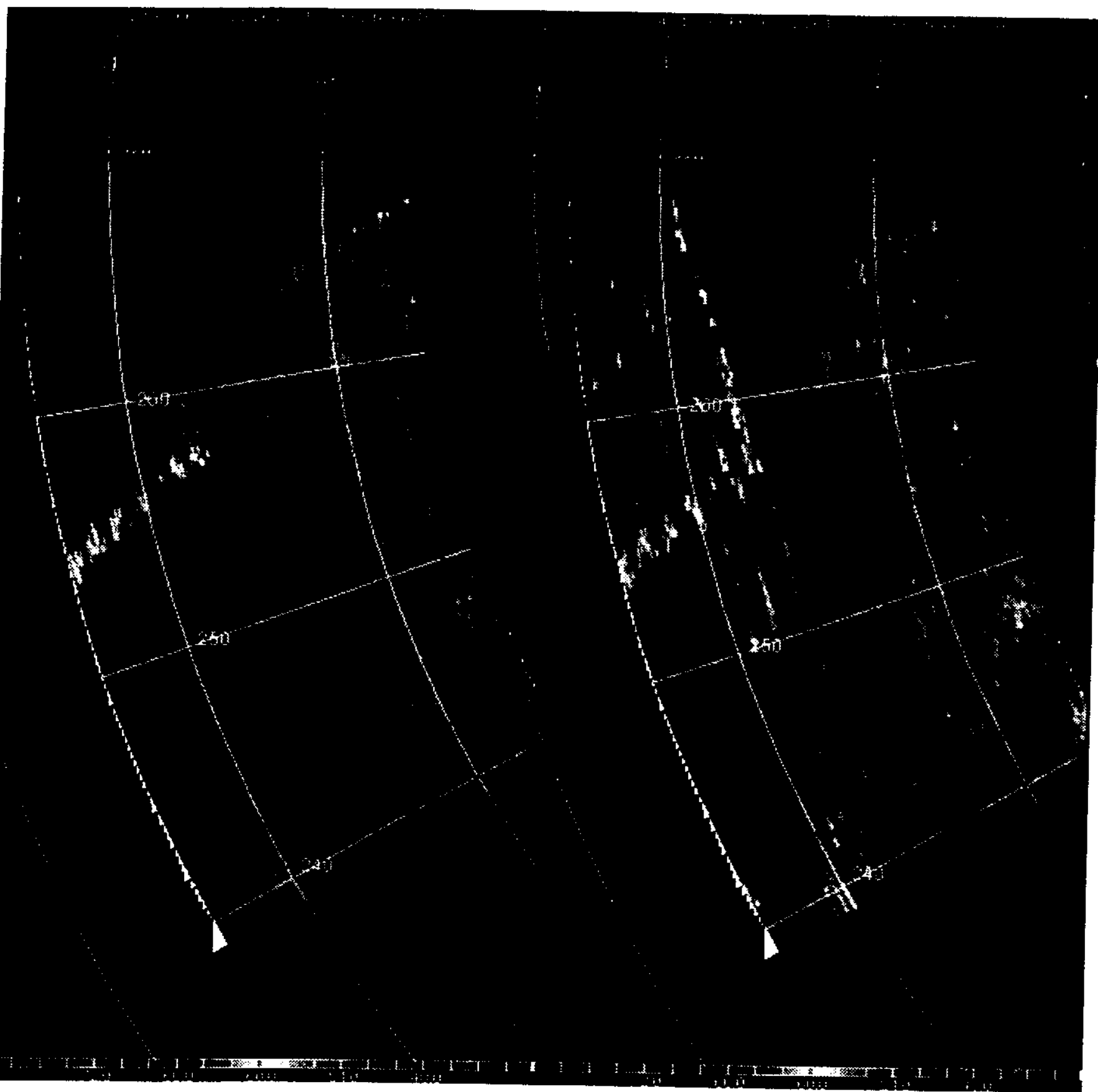

Fig. 2. Radar images of an IW packet preceding the packet in the fixed-beam observations shown in Fig. 1 . HH and VV images are in the right and left frames, respectively. 


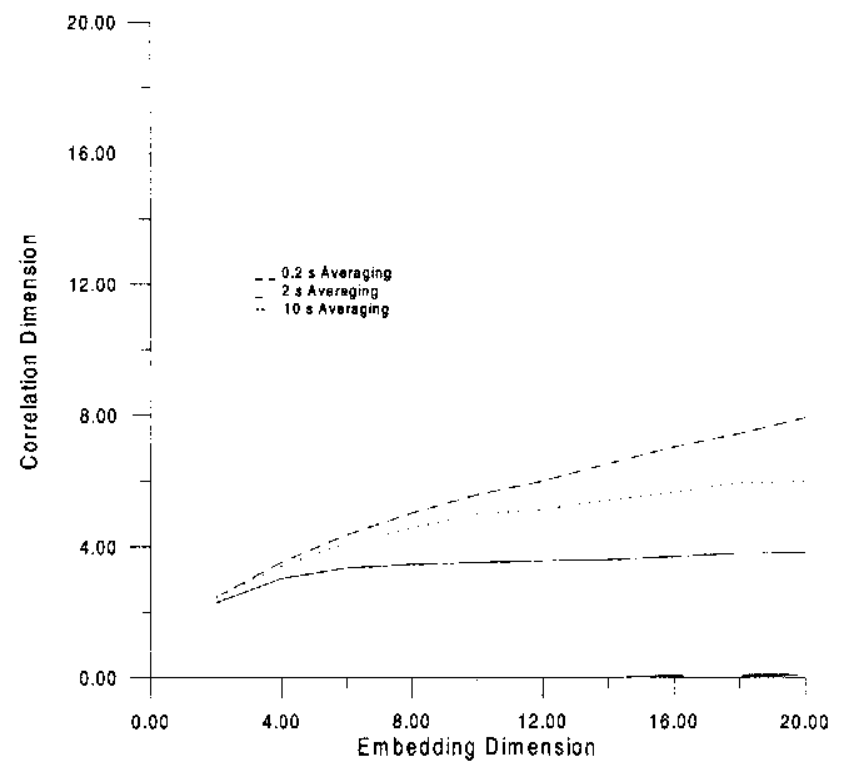

Fig. 3. Correlation dimension vs. embedding dimension for a 1,000 sample portion of the background radar backscatter time series (bcfore the arrival of the IW) for three different sampleaveraging times.

\subsection{Detection Results}

Following Haykin and Li, 1995, and Leung and Lo, 1993, we utilize prediction error for the radar return as our basic estimator for the detection algorithms. We train the algorithms on the portion of the radar backscatter time series before the arrival of the IW packet. This is the portion of the time series for which the correlation dimensions plotted in Fig. 3 were computed. $\Lambda$ s per the discussion above, inputs to the prediction algorithm are chosen to be six sequential time-averaged radar backscatter values. The algorithm is trained to predict the seventh value. Once trained, the algorithm was then applied to six sequential values of similarly averaged radar backscatter samples within the portion of time series containing the IW packet passage. A histogram is then formed of the algorithm prediction errors in both portions of the time series. Since the algorithm was trained on the portion of the time series beforc the arrival of the $I W$, the prediction errors resulting from applying the algorithm to the IW portion will generally be larger. A threshold prediction error can then be selected for declaring detection of the IW (more generally, for detecting a disturbance that is not sea clutter). The optimal threshold is usually chosen near the prediction error where the two histograms intersect.

Again following Haykin and Li, 1995, and Leung and Lo, 1993 , the two algorithms chosen to perform the prediction were statistical autoregression and an artificial neural network. The six autoregressive coefficients were determined by the Yule-Walker algorithm using the Fortran codes found in Maple, 1987. The neural network al-

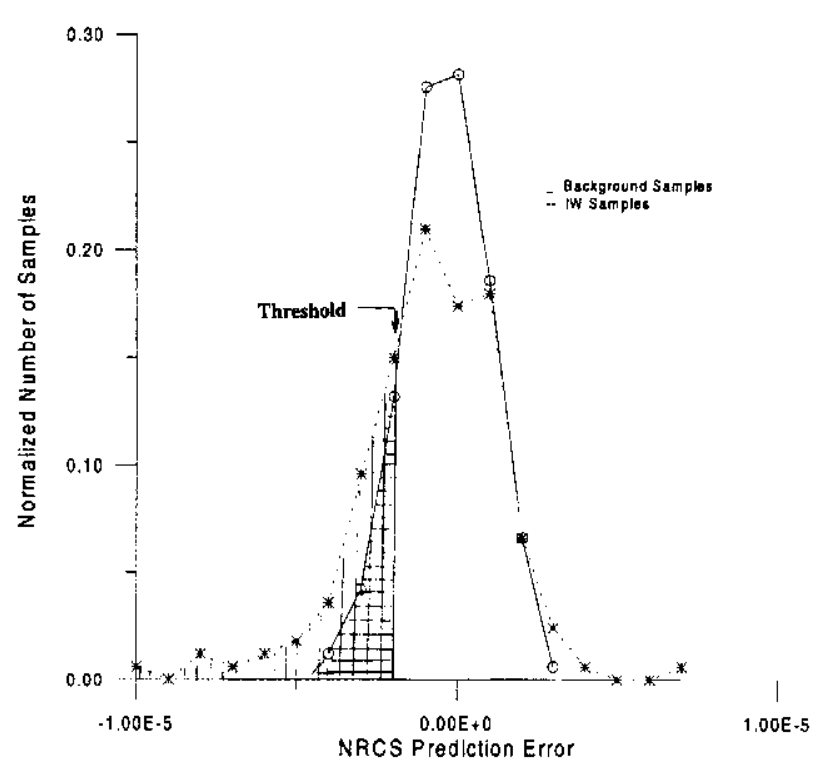

Fig. 4. Prediction error histogram for the background and IW portion of the radar backscatter time series block-averaged at $2 \mathrm{~s}$. The vertical hatching represents the probability of detection while the horizontal hatching represents the probability of false alarm.

gorithm chosen was a back- propagation known as Nets (Baffles, et.al., 1991), configured as a three layer perceptron. The single hidden layer of ten neurons was selected in accordance with guidelines found in the litcrature, (Johnson and Picton, 1996). In comparing the performance of these two algorithms, the regression coefficients and neural network weights were computed using the same background samples from the time series, and the same sets of input-output samples in the background and IW portions were used for prediction.

We conducted our detection comparisons as a function of sample averaging time for the radar backscatter time scries. In order to test the detection algorithms under conditions of weak IW signatures in the time series, and in order to have available a large number of samples in the five-hour time series, we limited the averaging times to intervals much less than the IW oscillation periods of near 10 min The number of independent examples used to train the algorithms and form the prediction error histograms ranged from 4250 for the 0.2 -s averaged samples to 45 for the 20-s samples. An example of the prediction error histograms for the neural network prediction using 2-s averaged samples is shown in Fig. 4. The histogram ordinate is normalized so that the total number of prediction errors is unity. Thus, once a prediction error threshold is specified, the probability of detection and probability of false alarm can be represented by the sums under the respective areas indicated in Fig. 4. Finally, the receiver operating curve (ROC) for probability of detection vs. probability of false alarm is plotted by allowing the prediction error threshold to range over all of the prediction error values. The ROCs 


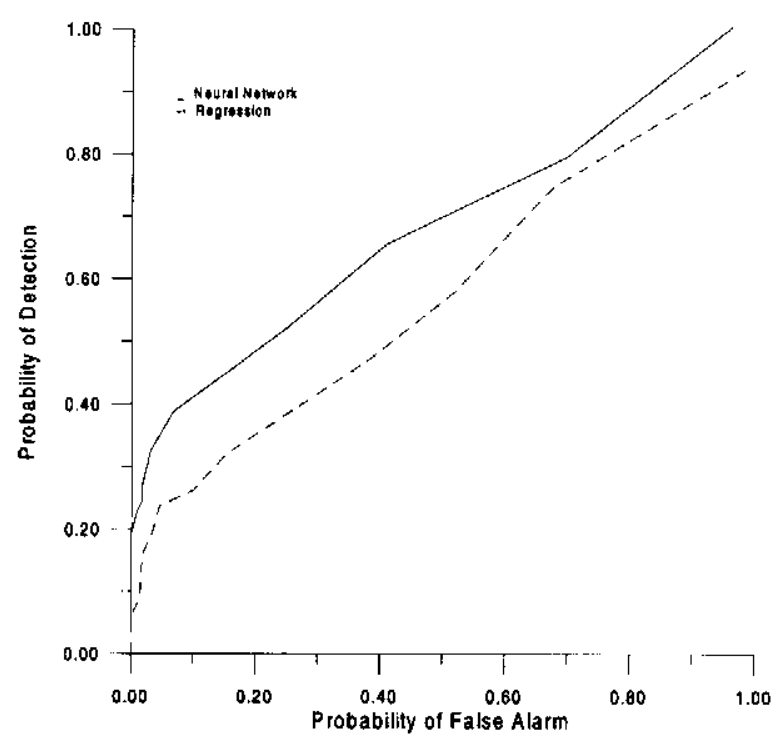

Fig. 5. Receiver operating characteristics for the neural network and statistical regression detection algorithms for the 2-s sample averaging time.

for IW detection by the regression and neural network are presented in Fig. 5 for the 2-s averaged data. The neural network is seen to outperform regression.

Rather than plot separate ROCs for each sample averaging time used, we plot the difference in rms prediction error for the background and IW portions of the time series as a function of averaging time. It is clear from the above discussion that detection performance, as measured by the ROCs, will be greater as this difference increases. The normalized rms prediction error difference (normalized by the rms prediction error for the background) is plotted in Fig. 6 for both the neural network and regression predictions.

\subsection{Discussion of Detection Results}

It is generally expected that detection of weak signals within a background process that exhibits signatures of low-dimensional chaos will benefit from processing the background as a deterministic rather than a random process. This expectation was confirmed for radar detection of sea ice within a sea clutter background (Haykin and Lo, 1995, and Leung and Li, 1993), and was confirmed here for radar detection of internal waves within a sea-clutter background. In both cases, the order of the detection algorithms was chosen to be near the correlation dimension found for the background. We stress that here and in Haykin and Lo, 1995, and Leung and $\mathrm{Li}, 1993$, the evaluations compare a statistical and deterministic method for processing the background sea clutter in the detection of a disturbance which is not sea clutter. Clearly, in order to distinguish one type of disturbance from another, say sea ice from an internal

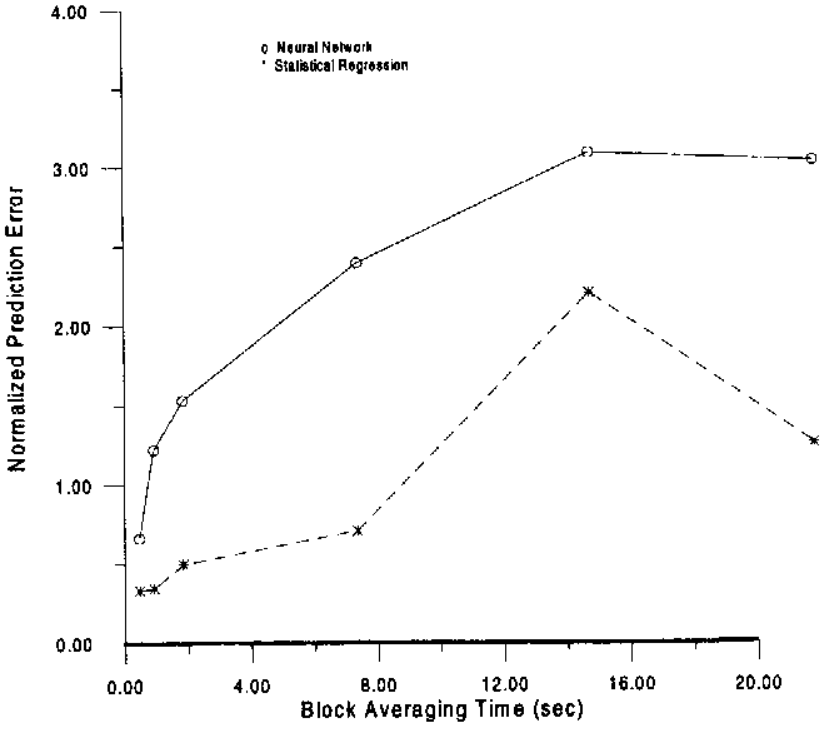

Fig. 6. Normalized rms prediction error difference between the IW and background portion of the radar backscatter time series vs. sample averaging time.

wave, one needs to combine the optimal deterministic processing of the background with additional processing such as spectral filtering which can isolate signatures specific to the disturbance of interest.

The detection performance of both the ncural network and regression algorithms is seen in Fig. 6 to decrease at the longer averaging times. This is probably the result of the smaller number of training samples for the algorithms. The reduced prediction errors for both the neural net and regression algorithms at the shorter averaging time is due to the smaller dynamical signature of the 10-min period IWs in this regime.

Of primary interest is the degradation of performance of the deterministic algorithm relative to the statistical processing at shorter averaging times. We hypothesize that this is caused by the introduction of short time scale variability into the background that is generated by higher dimensional dynamics on the surface. This interpretation is supported by the correlation dimension results in Fig. 3 which show a lack of saturation for the 0.2 -s averaging-time curve. The time scale of this postulated high dimensional variability is thus between $0.2-2.0 \mathrm{~s}$. This is a much shorter time scale than the high dimensional surface variability found for the studies reported below, and unlike that case, the variability is not revealed in either the time series Fourier transform or in range-time radar images of the surface.

\section{Wind and Stress Retrieval}

The possibility of developing deterministic algorithms for retrieving wind and stress over the oceans from sea- 
surface, grazing-angle radar backscatter first became apparent to us after analyzing a six-hour-long time series of measured horizontal ocean surface winds and a concurrently measured time series of grazing angle, vertically polarized radar backscatter returns from an area of sea surface near the site where the winds were measured, (Palmer et al., 1995). Our analysis strongly suggested that the variability of both time series on time scales of order $13 \mathrm{~s}$ was governed by the same dynamical system, and that the number of coupled degrees of freedom present in the dynamical system governing this variability was as small as six. Here, we report new results and interpretations for this same data set relevant to developing deterministic algorithms for retrieving surface wind and stress from grazing angle radar backscatter.

As before, our approach is directed towards exploiting dynamical signatures in the data, i.e. developing an algorithm based deterministic temporal patterns, rather than statistical moments. This type of algorithm differs fundamentally from the algorithm most commonly used to retrieve wind and stress from ocean surface radar backscatter algorithm. This latter algorithm is a two parameter empirical formula relating time-averaged wind or wind-stress to the time- averaged radar backscatter cross-section (Schroeder, et al, 1982; Geernaert and Keller, 1992. Besides being designed for temporal averages this algorithm is also fit to radar backscatter data at much smaller incidence angles than the grazing angle data used here, so we do not make performance comparisons with it here.

\subsection{SCOPE Ocean Sensing Experimient}

The radar-backscatter and surface-wind data used for this study were obtained as part of an experiment similar to the COPE experiment used for the IW detection studies above. The experiment, termed The San Clemente Ocean Probe Experiment (SCOPE), was performed in September 1993 by the NOAA Environmental Technology Laboratory (ETL) near San Clemente Island, $100 \mathrm{~km}$ from San Diego. The radar measurements were obtained with an X-band coherent radar located $573 \mathrm{~m}$ above the sea surface on San Clemente Island. The surface winds were obtained from an atmospheric surface-layer sensing package developed at NOAA/ETL (Fairall, et al, 1996) and placed aboard the Scripps Institute Floating Instrument Platform (R.V.FLIP), which was located $31 \mathrm{~km}$ upwind from the radar. Additional details on the experiment can be found in Palmer, et al 1995, and Kropfli and Clifford, 1994.

The three measured time series used for this analysis are shown in Fig. 7. They are the VV polarized radar backscatter, the scalar horizontal wind-speed, $\mathrm{U}$, and the scalar wind stress, $\langle U w\rangle$ where $\mathrm{w}$ is the vertical wind, and $\langle>$ indicates a coherent average. As in the IW detection study, two nonlinear dynamics tools are used to analyze the data: the correlation dimension, and an artificial neural-network, with the network using six input neurons to accept six sequential samples of radar backscatter values to correspond with a sixdegree-of-freedom dynamical system suggested by the correlation-dimension analysis (Palmer, et.al, 1995; Elsner and Tsonis, 1989). A single output neuron was chosen to correspond to a single retrieved value of wind or stress. Again, a single hidden layer of ten neurons was selected in accordance with guidelines found in the literature. (Johnson and Picton, 1996).

\subsection{Correlation Dimension}

In Palmer, et.al, 1995, we presented the correlation dimension for five measured time series, each blockaveraged into $13 \mathrm{~s}$ samples. The time series were measured concurrently in SCOPE during a six-hour period in which the radar was pointing toward FLIP. The fact that a saturation in the correlation dimension was seen at nearly the same value for both the vertically polarized radar backscatter time series and the horizontal surface wind-speed time series, and was not seen for the other time series suggested that there is a single dynamical system governing these two observables with an attractor dimension near six. Our interpretation of why the same low correlation dimension was not found for the horizontally polarized reflectivity and the Doppler signal was that these observables are probing the higher dimensional dynamical system governing the longer ocean-surface waves. On the other hand, it was puzzling that the vertical winds did not exhibit the same correlation dimension as the horizontal winds. They are clearly part of the same boundary layer dynamical system that governs the horizontal winds.

One possible explanation for higher vertical wind correlation dimension lies in the fact that the turbulence is not locally isotropic. Evidence for this can be found in the average velocity spectra shown in Fig. 8 . In the vertical axis we show the average of 4550 -min realizations of the three velocity components (stream-wise, crossstream and vertical) using the Kaimal et al.,1972 normalization, which causes the data to collapse to a universal form in the inertial-sub-range of locally isotropic turbulence (see Panofsky and Dutton, 1984 for more detailed discussion). In the inertial sub-range the variance spectra exhibit the classic $-5 / 3$ frequency power dependence (in this graph $\mathrm{f}^{*} \mathrm{~S}$ obeys a $-2 / 3$ power law) and the ratio of the transverse to stream-wise spectra is expected to be $4 / 3$. We can see that this ratio is obeyed for $f z / u>5$. Note that for frequencies below the isotropic range, the variance contained in the horizontal components continues to increase (almost following the $-2 / 3$ slope) while the vertical component levels off and even begins to decrease with decreasing frequency. This is because the larger eddies responsible for this variability cannot have significant vertical amplitude near the surface. In this lower frequency range, the horizontal ve- 

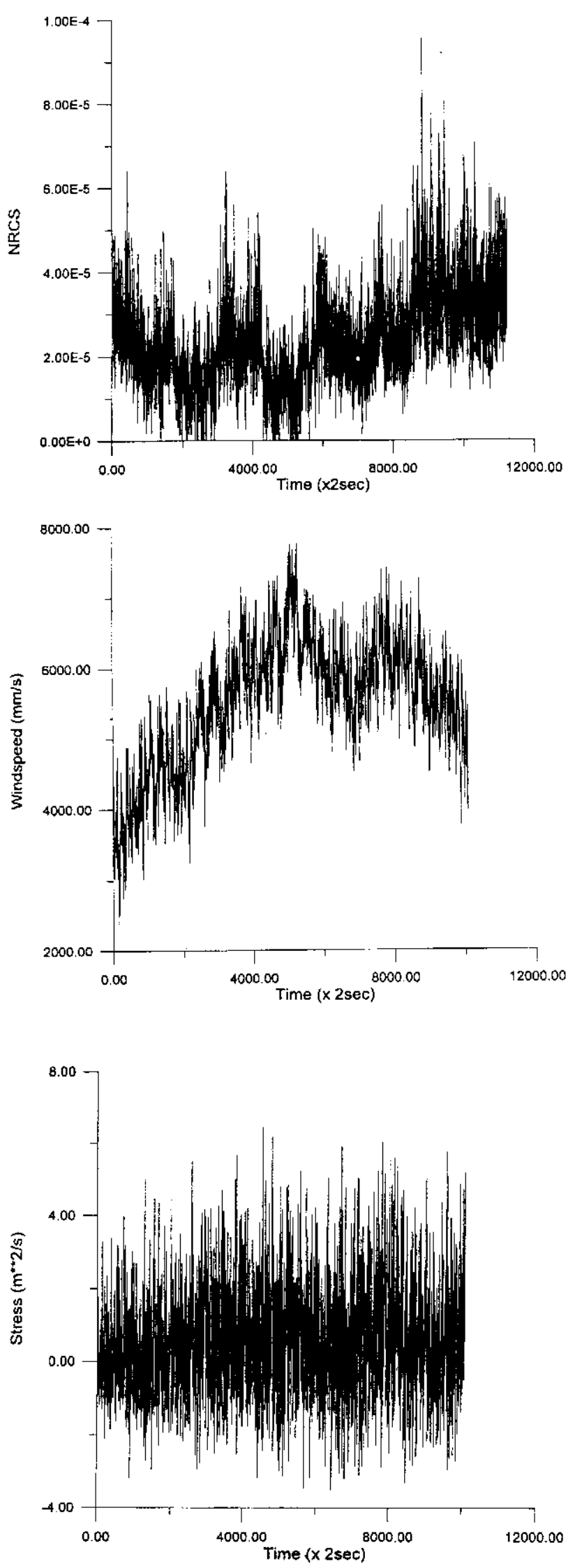

Fig. 7. Time series data used for the analysis. a: VV polarized, normalized radar cross section per unit area (NRCS). b: Horizontal wind speed. $c$ : Wind stress.

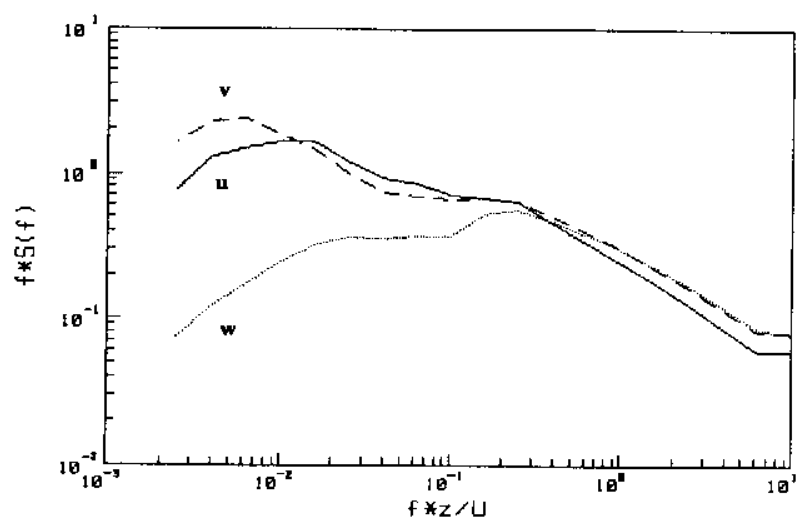

Fig. 8. Three velocity component spectra normalized as per Kaimal et al. [1972]; u=streamwise, $v=$ cross-stream, and $\mathrm{w}=\mathrm{vertical}$. The vertical axis is the frequency times the variance spectral intensity divided by $u^{*} 22 / 3$ where $u^{*}$ is the friction velocity (square-root of the w-u covariance) and the stability-dependent dimensionless turbulent kinetic energy dissipation rate. The lines represent the average of $4550-\mathrm{min}$ samples for a stability range corresponding to the data analyzed in this paper. The horizontal axis is the dimensionless frequency, $\mathrm{fz} / \mathrm{U}$, where $\mathrm{f}$ is the frequency $(\mathrm{Hz}), \mathrm{z}$ the height of the measurement $(\mathrm{m})$, arid $\mathrm{U}$ the mean wind vector magnitude (ms-1). The flattering of the curves at the higher frequencics represents the noise level of the sonic anemometer.

locity components tend to continue to exhibit "inertial" behavior (i.e., a cascade of large scales to smaller) but the vertical component does not. The wind measurements used in this study were obtained on FLIP at a height of $10 \mathrm{~m}$ above the surface, and were taken during a period when the wind speed averaged about $5 \mathrm{~m} / \mathrm{s}$. Thus, the block-averaging interval of $13 \mathrm{~s}$ used in the earlier analysis corresponds to sampling the data in the inertial sub-range for the horizontal winds, but outside the inertial sub-range for the vertical winds. The impact of this for our dynamical study is not so much the attenuation of the vertical winds in this regime as it is the introduction of another dynamical system contributing to the measured vertical wind variability, namely, that of the long ocean waves.

Below, we present results for the computed correlation dimension for the three time series for block averaging times of the data equal to $2 \mathrm{~s}$ and $20 \mathrm{~s}$. The correlation dimension is computed for a 1000-point time series in all cases. This means that different regions of the time serics are sampled for the two correlation-dimension computations. While the raw timle series shown in Fig. 7 are clearly non-stationary, the computed correlation dimensions appear to be reasonably invariant to the region of the time series from which points are selected for a fixed block averaging time. An example of this invariance is shown in Fig. 9 for the horizontal wind correlation dimension computed at the $2 \mathrm{~s}$ averaging time. Thus, we feel confident that the differences in correlation dimensions found below are not dominated by the non-stationarity of the data. 


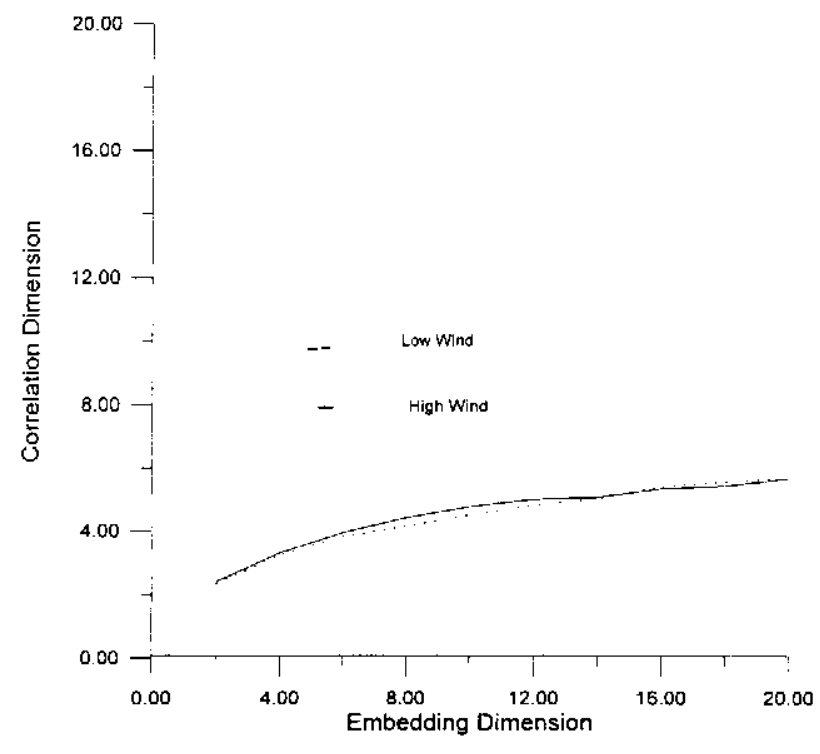

Fig. 9. Horizontal wind correlation dimension vs. embedding dimension computed for the 2 -s averaging time at an early, 1000point, low-wind region of the time series and at a later 1000-point, higher wind region.

\subsubsection{Stress Correlation Dirnension}

Since the stress is a product of vertical wind and horizontal wind, we expect that it will fail to exhibit a saturated correlation dimension at the 20 -s averaging interval for the reasons cited above for the vertical wind. However, according to the above argument, we would also expect the stress time series to begin to exhibit a correlation dimension comparable to that of the horizontal winds when sampled in the inertial sub-range. This was indeed found to be the case as demonstrated in Fig. $10 \mathrm{a}$, which shows the stress correlation dimension as a function of the embedding dimension for two different block-averaging times for the data. The insel show the stress spectrum.

\subsubsection{Horizontal Wind Correlation Dimension}

Fig. 8 shows that unlike the vertical wind, the horizontal wind will remain measured in the inertial subrange for both a 2-s and 20-s block-averaging interval. Fig. 10b shows the horizontal wind correlation dimension computed for these two averaging times, and the wind spectrum in the inset. As expected, the computed correlation dimension does not depend sensitively on the averaging interval in this case.

\subsubsection{Radar Backscatter Correlation Dimension}

Fig. 10c shows a significant difference in the computed correlation dimension for the radar backscatter time series for the two averaging times, but in an opposite sense from that of the wind stress. This is the same behavior
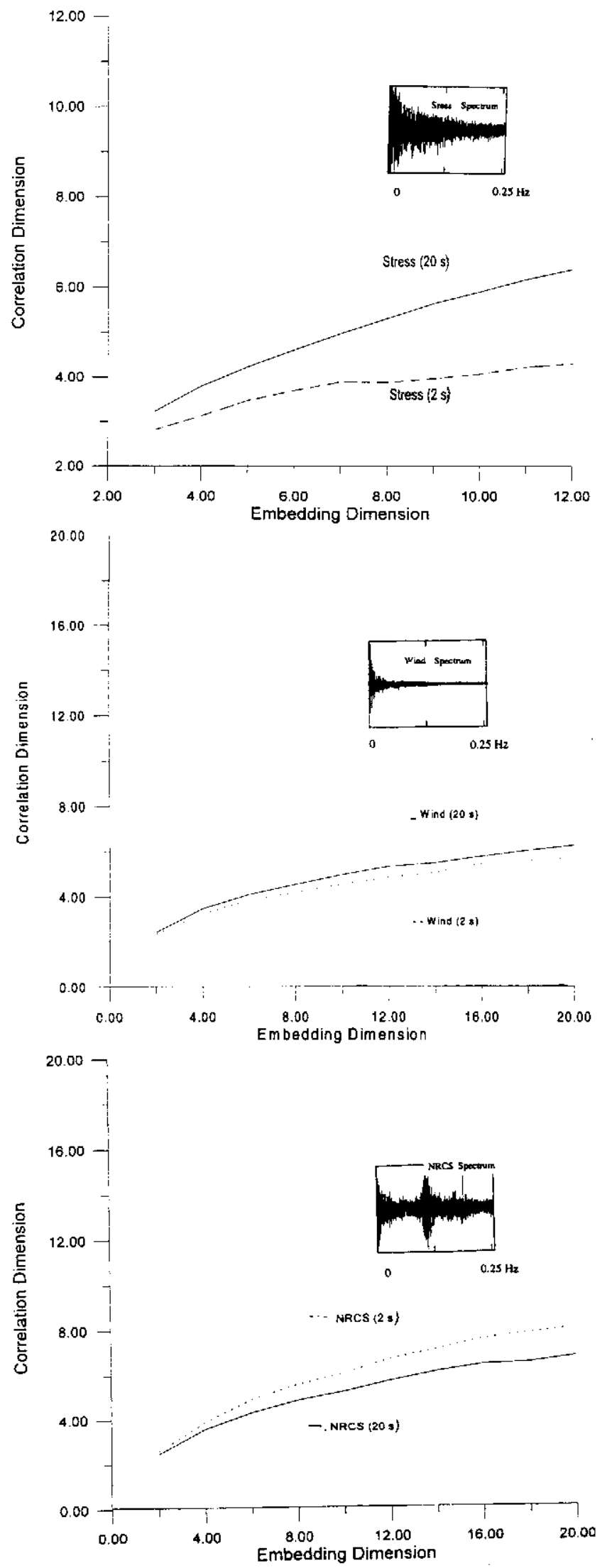

Fig. 10. Correlation dimension vs, embedding dimension for 2-s and 20 -s block averaging times for a 1000-point time series and the Fourier transform of the 2-s sampled, time series, a: Wind stress. b: Horizontal wind. c: VV-polarized radar backscatter (NRCS). 
that was found for the IW detection radar data (Sect. 2.4 ), and our interpretation is the same: It is a result of sampling a higher dimensional dynamical system associated with the long waves. The longer 20-s averaging time filters out most of the long wave variability, but leaves the variability associated with the lower dimension dynamical system governing the wind driven radar backscatter. In this case, additional support for this interpretation can be found in the radar backscatter spectrum shown in Fig. 10c and the range- time image of radar data shown in Fig. 11. The spectrum and particularly the image clearly shows the presence of the long surface wave variability in the radar backscatter.

\subsection{Retrieval Results}

Fig. 12 shows the rms error for retrieving the measured horizontal wind and stress from the radar backscatter time series using the above-described neural network with six sequential, block-averaged samples of the radar backscatter as input. The retrieval error is plotted as a function of block-averaging time for the data. The wind or stress value that the neural net was trained to retrieve (the neural-net output) was the block-average wind or stress value occurring immediately following the six input block-averaged radar backscatter samples. The number of input-ouput training examples was kept constant at 83 for all of the averaging times used. The number of training cycles used was limited to 10,000 . Finally, the rms errors presented are for scaled values of the measured and neural net output wind and stress values and thus have relative significance only.

Our final result is a computation of normalized rms errors for retrieving wind and wind-stress with the neural net as a function of average wind speed. The net was trained on the last half of the three measured time series, and its retrieval performance was computed on the first half. This allowed the results to be presented as a function of average wind-speed since the wind-speed increased throughout the firsi half. The retrieval performance is again characterized with the rms prediction error, but in this case the errors presented are unscaled values normalized by the measured value. The block averaging time used was $20 \mathrm{~s}$, and the number of neural net training cycles used was again limited to 10,000 . These results are shown in Fig. 13. There is a suggestion of increasing retrieval error in the lower windspeed regime, as occurs also for the statistical retrieval algorithm (Geernaert and Keller, 1992).

\subsection{Discussion of Retrieval Results}

As measured by the rms error, the performance of the neural net in retrieving the wind values is seen to generally improve as the block-averaging time is increased. This is consistent with the correlation dimension result found for the radar and for the horizontal wind, which indicated a lowering of the radar time-series correlation dimension in going to the longer block- averaging time, and a relative insensitivity of the wind correlation dimension to averaging time. The longer the averaging times, the less variability is present due to the long waves, and the better the neural net is at functioning as a nonlinear transfer function for the low-dimensional dynamical system governing the wind-driven radar reflectivity. On the other hand, the stress retrieval exhibits an optimal averaging time where a minimum rms crror is achieved. The interpretation for this behavior is based on the above observation that the correlation dimension computed for the wind stress time series varied in an opposite sense to that for the radar reflectivity time series. The optimal neural net performance for retrieving the measured wind stress evidently occurs at an averaging time that minimizes the intrusion of the long surface wave variability in the combined radar and wind-stress time series as utilized by the neural net.

\section{Conclusions}

We have examined radar backscatter data from one ocean sensing experiment and coincidently measured radar backscatter and surface wind data from another ocean sensing experiment with two nonlinear dynamic analysis tools, the correlation dimension and an artificial neural network. The correlation dimension was used to estimate the minimum number of significant dynamical degrees of freedom responsible for the temporal variability of the data obtained at a fixed location on the sea surface. The neural network was used to generate a nonlinear response function that was applied to radar detection ocean internal waves, and to generate a nonlinear transfer function applied to radar retrieval of surface winds and stress. In both of these applications, the performance of the neural network algorithms with a fixed number of input and hidden neurons $(6$ and 10 respectively) was found to improve when the data was block averaged to produce a minimum value for the correlation dimension at a fixed embedding dimension. We hypothesize that this behavior is caused by the presence of two weakly coupled dynamical systems. The lower dimensional system governs the surface winds that drives the surface structure responsible for the radar backscatter (principally centimeter-scale Bragg-structure) on time scales within the wind turbulence inertial sub-range, and exhibits correlation dimensions of 4-6. The other, higher dimensional dynamical system is assumed to govern the longer surface waves which modulate the radar backscatter. Nonlinear dynamic analysis of pressure sensor measurements of ocean surface waves indicate a dynamical attractor dimension of 6 or greater (Frison and Abarbanel, 1997; Elgar and Mayer-Kress, 1989). In the case of the wind and stress retrieval application, additional support for the hypothesis comes from the observation 


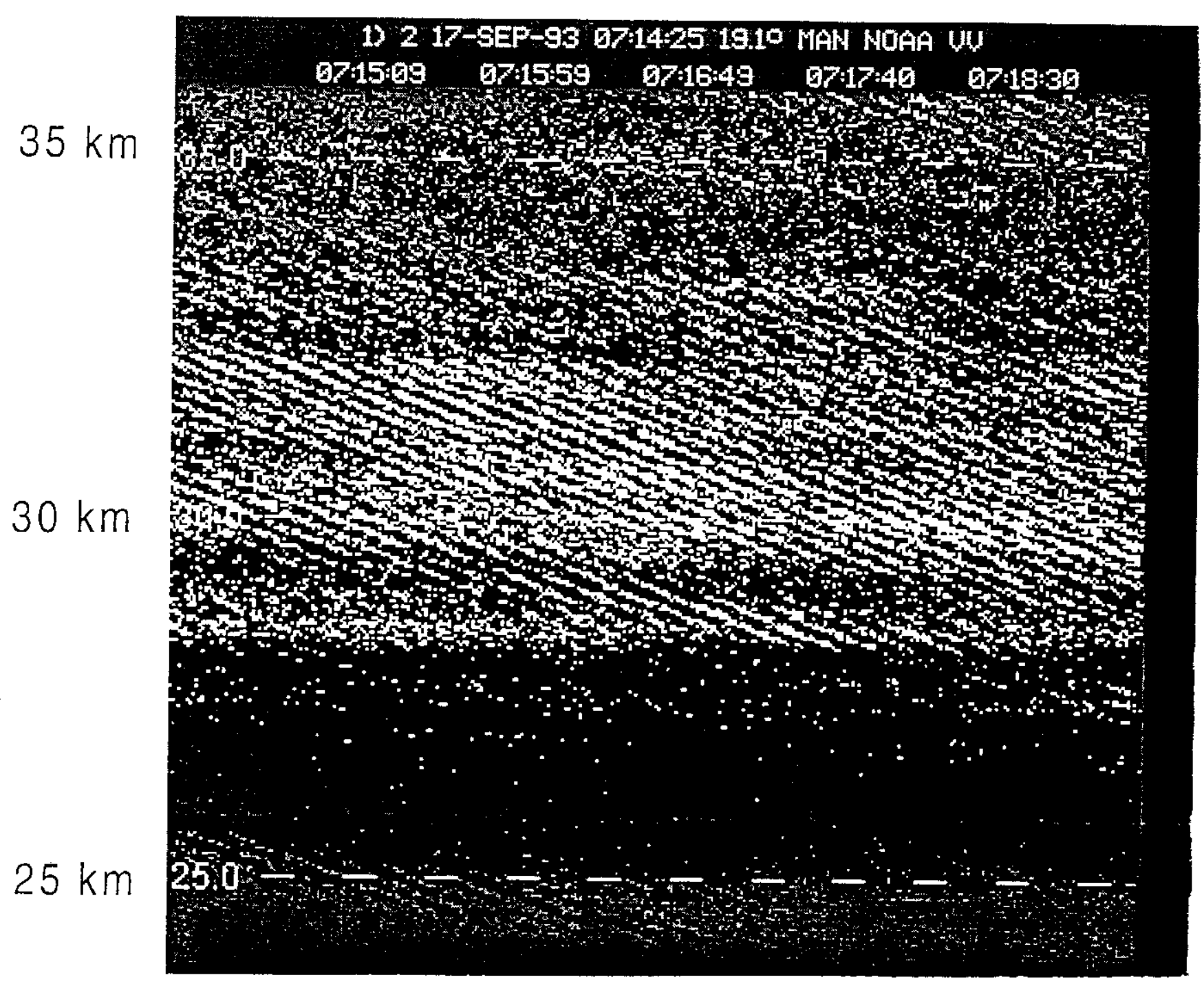

Fig. 11. Range-time image of the radar backscatter data used in the analysis showing the presence of a $10 \mathrm{~s}$ period surface-wave field. 


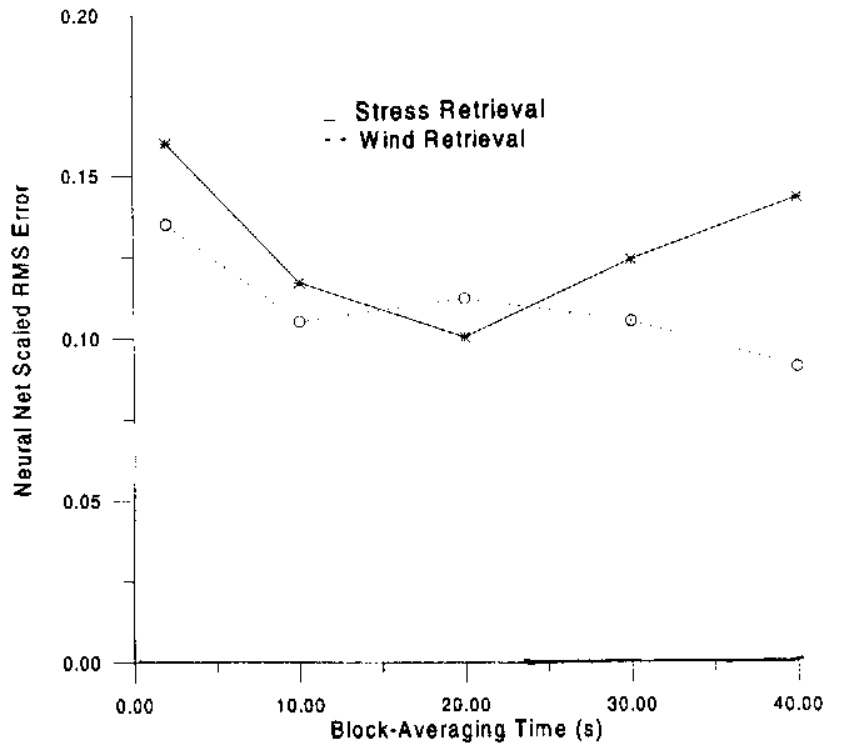

Fig. 12. Scaled rms errors vs. block-averaging time for windspeed and stress magnitude retrieval from NRCS using the neural network algorithm.

of long wave variability in range-time images and temporal Fourier transforms of the radar backscalter data.

The above analysis was conducted at time scales ranging from $0.2-20 \mathrm{~s}$. Previous observations of deterministic chaos in radar backscatter from the sea-surface were obtained at time scales of a few milliseconds (Haykin and $\mathrm{Li}, 1995$, and Leung and Lo, 1995), where indicated correlation dimensions ranged from $6.5-9$. The dynamical system responsible for this very short time scale variability is unknown, and is probably strongly decoupled from the wind and surface wave dynamical systems probed in this work.

In summary, we have found further evidence that the $10 \mathrm{~s}$ scale temporal variability of grazing angle radar backscatter from a fixed location on the ocean surface is caused by a low dimensional dynamical system that governs the surface-wind turbulence. We have also found evidence that this low dimensional backscatter signal can be "contaminated" by a higher dimensional system governing the surface wave dynamics, and that the contamination can be removed by block averaging of the data. This averaging procedure improved the performance of neural network algorithms with 6 input neurons trained for grazing angle radar detection and retrieval applications. Much work remains in order to fully understand and define these two nonlinear dynamical systems, but our results shed some light on the time scales where coupling between the two systems occurs and the implications that this coupling has to the development of deterministic algorithms for radar delection and retrieval applications.

Acknowledgements. The authors thank Ann Keane for her help in processing the radar data, and the team of ETL scientists, en-

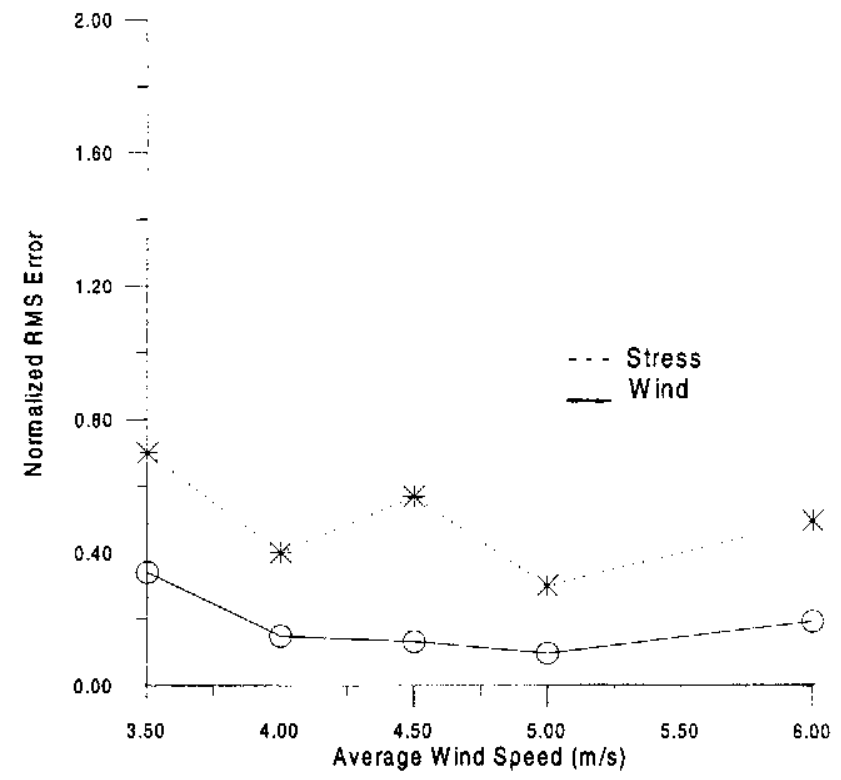

Fig. 13. Normalized neural net rms retrieval errors vs. average wind speed for $20 \mathrm{~s}$ block- averaging time.

gineers, and technicians who helped gather the radar and in-situ data. 'This work was supported by the DoD Advanced Sensor Application Program and the ONR Marine Boundary Layer program.

\section{References}

Baffles, P.T., R. O. Shelton, and T. A. Phillips, NETS Users Guide, NASA Johnson Space Flight Center. Available from COSMIC, University of Georgia, 382 East Broad St., Athens, GA 30602,103, 1991.

Ding, M., C. Grebogi, E. Ott, T. Sauer, and J. York, Plateau Onset for Correlation Dimension: When does it Occur?, Phys. Rev. Lett., 70, 3872-3875, 1993.

Elgar, S. and G. Mayer-Kress, Observation of the fractal dimension of deep and shallow water ocean surface gravity waves, Physica D 37, 104, 1989.

Elsner, J. and A. Tsonis, Nonlinear Prediction, chaos, and noise, Bull. Amer. Meteor. Soc., 73, 49-60, 1992.

Frison, T.W. and H.D.l. Abarbanel, Ocean gravity waves: A nonlinear analysis of observations, J. Gcoph. Res. 102, 1051-1059 (1997).

Fairall, C.W. and A.B. White, Integrated Shipboard Measurcments of the Marine Boundary Layer, J. Ocean Atmos. Technol., Accepted, 1996.

Geernaert, G.L, and W.C. Keller, On the wind power law and atmospheric stability effects in occan scatterometry, ISPRS Journal of Photogrammetry and Remote Sensing. 47, 369-386, 1992.

Grassburger, P. and I. Procaccia, Characteristics of strange attractors, Phys. Rev. Lett, 50, 346-349, 1983.

Haykin, S, and X. B. Li, Detection of signals in chaos, Proc IEEE, $83,95-122,(1995)$.

Johnson, J. and P. Picton, How to train a neural network, Complexity, 3, 13-28, 1996.

Kaimal, J. C., J. C. Wyngaurd, Y. Izumi, and O. R. Cotc, Spectral characteristics of surface layer turbulence, Quart. J. Roy. Met. Soc. 98, 563-589, 1972.

Kropfli, R.A. and S. F. Clifford, The San Clemente ocean probing 
experiment: A study of air-sea interactions with remote and in-situ sensors, Proceedings of the IGARSS Conference, 1994.

Kropfli, R.A. and S. F. Clifford "The coastal ocean probing experiment: Further studies of air- sea interaction with remote and in-situ sensors", Procecdings of the IGARSS Conference, 1996.

Leung, H. and S. Haykin, Is there a radar clutter attractor?, Appl. Phys. Lett. 56, 593 595 (1990).

Leung, H. and T, Lo, Chaotic radar signal-processing over the sea, IEEE J. Oceanic Eng. 18, 287-295, 1993.

Maple, S.L., Digital spectral snalysis with applications, PrenticeHall, New Jersey, 1987.

Mityagina, M.I., V. G. Pungin, A. V. Smirnov, and V. S. Etkin, The changes in the power-carrying part of the sea surface wave spectrum in a field of internal wave according to remote observation, Izv. Atmos. Ocean Phys., 27, 925-929, 1991.

Palmer, A.J., R.A. Kropfli, and C.W. Fairall, Signatures of deterministic chaos in radar sea clutter and ocean surface winds,
Chaos, 5, 613-616, 1995.

Panofsky, H. A., and J. A. Dution, Almospheric Turbulence, Wiley-Interscience, New York, pp 397, 1984.

Schroeder, L.C., D. H. Boggs, G. Dome, I. M. Halberstam, W. L. Jones, W. J. Pierson, and F. J. Wentz, The relationship between wind vector and normalized radar cross section used to derive SEASAT-A Satellite Scalterometer Winds, J. Geophys Res, 87, 3318-3336 1982.

Smirnov, A.V. and V.U. Zavorotny, Study of Polarization Differences in Ku-Band Ocean Radar Imagery, J. Physical Oceanography, 25, 2215-2228, 1995.

Thiria, S., C. Mejia, F. Badran, and M. Crepon, A Neural Network Approach for Modeling Nonlinear 'Iransfer Functions: Application for wind Retrieval from spaceborne scatterometer data, J. Geophys. Res., 98, 22, 827-841, 1993.

Wetzel, I.B., Electromagnetic scattering from the sea at low grazing angles, in Surface Waves and Fluxes, Vol 1I, 109-172, G.L. Gccrnacrt and W.J. Plant Eds., Kluwer Academic Press, 1990. 